\title{
RELATIVE INTEGRAL BASES
}

\author{
LAWRENCE C. WASHINGTON
}

ABSTRACT. We give necessary and sufficient conditions for certain quadratic extensions of imaginary quadratic fields to have relative integral bases.

Two recent papers ([2], [4]) have given examples of quadratic extensions of imaginary quadratic number fields for which there exists no relative integral basis. In this note, by using a theorem of Artin, we prove the following theorem, which contains these examples as a special case (namely, when $|a|$ is prime).

TheOREM. Let $F=\mathbf{Q}(\sqrt{ }-D)$ be an imaginary quadratic field with discriminant $-D$. Let $a \equiv 1(\bmod 4)$ be a square-free integer with $D \nmid a$, and let $E=\mathbf{Q}(\sqrt{ }-D, \sqrt{ } a)$. Then $E / F$ has a relative integral basis $\Leftrightarrow(a, D)=1$.

Proof. Let $g=(a, D)$, so $g$ is odd and square-free. The quadratic subfields of $E / \mathbf{Q}$ have discriminants $a,-D$, and $-a D / g^{2}$. Therefore, up to sign, these are the conductors of the nontrivial Dirichlet characters for $E / \mathbf{Q}$. By the conductor-discriminant formula, $D_{E / \mathbf{Q}}=$ discriminant of $E / \mathbf{Q}=\left(a^{2} D^{2} / \mathrm{g}^{2}\right)$, regarded as an ideal of $\mathbf{Z}$. Letting $D_{E / F}=$ relative discriminant for $E / F$, we have

$$
D^{2} N_{F / \mathbf{Q}}\left(D_{E / F}\right)=D_{E / \mathbf{Q}}=\left(a^{2} D^{2} / g^{2}\right) .
$$

Therefore, $N_{F / \mathrm{Q}}\left(D_{E / F}\right)=\left(a^{2} / g^{2}\right)$. If $\sigma$ restricted to $F$ generates $\mathrm{Gal}(F / \mathbf{Q})$, then $\sigma\left(D_{E / F}\right)=D_{\sigma E / \sigma F}=D_{E / F}$. Consequently, $D_{E / F}^{2}=N_{F / Q}\left(D_{E / F}\right)=(a / g)^{2}$, so $D_{E / F}=(a / g)$.

The polynomial $X^{2}-a$ generates $E / F$, and the discriminant $\Delta$ of this polynomial is $4 a$. Therefore, $\Delta^{-1} D_{E / F}=(g) / 4 g^{2}$.

We now need the following theorem of Artin ([1]): Let $E / F$ be a finite extension, $D_{E / F}$ the relative discriminant, and $\Delta$ the discriminant of a polynomial defining $E / F$. Then $E / F$ has a relative integral basis if and only if there is a principal ideal $(z)$ of $F$ such that $(z)^{2}=\Delta^{-1} D_{E / F}$.

Consequently, our problem is reduced to determining when there exists $z \in F$ such that $(z)^{2}=(g)$.

If $g=1$, let $z=1$. We find that $E / F$ has a relative integral basis. In fact

Received by the editors April 14, 1975.

AMS (MOS) subject classifications (1970). Primary 12A99; Secondary 12A25, 13 B20.

Key words and phrases. Relative integral bases, quadratic fields. 
the basis for $Q(\sqrt{ } a) / Q$ is also a basis for $E / F$ (see Lang [3, p. 68]).

If $g \neq 1$ we have two cases:

(i) $-D \not \equiv 1(\bmod 4)$. In this case $z=x+y \sqrt{ }-D$, with $x, y \in \mathbf{Z}$. Taking norms, we obtain $g^{2}=N\left(z^{2}\right)=\left(x^{2}+D y^{2}\right)^{2}$, hence $g=x^{2}+D y^{2}$. Since $D \nmid a, g<D$. Therefore, $y=0$. But $g$ is square-free, so $g \neq x^{2}$. Therefore, $z$ does not exist.

(ii) $-D \equiv 1(\bmod 4)$. In this case we obtain $g=x^{2}+x y+(1+D) y^{2} / 4$, with $x, y \in \mathbf{Z}$. Since $g$ is square-free, $y \neq 0$. Therefore, $g \geqslant D y^{2} / 4>g y^{2} / 4$, so $y= \pm 1$; we also find that $4 g \geqslant D>g$. Since $D$ is a multiple of $g$ and $D$ is odd, $D=3 g$. Consequently, $g=x^{2} \pm x+(1+3 g) / 4$, which implies $g=(2 x \pm 1)^{2}$, contradiction. Therefore, $z$ does not exist. Q.E.D.

In the cases considered by MacKenzie, Scheuneman, and Fujisaki, $|a|$ is a prime which divides $D$, so $E / F$ is unramified; in fact, $E$ is contained in the genus field of $F$. One may ask more generally whether or not $E / F$ has a relative integral basis, where $E$ is any subfield of the genus field of $F$. It turns out that the above case is an exception and that usually a basis exists.

THEOREM. Let $F=\mathbf{Q}(\sqrt{ } d)$ be a quadratic number field with discriminant $d$, let $\left\{p_{1}, \ldots, p_{n}\right\}$ be a proper subset of the set of odd primes dividing $d$, and let $E=F\left(\sqrt{ \pm p_{1}}, \ldots, \sqrt{ \pm p_{n}}\right)$, where $\pm p_{i} \equiv 1(\bmod 4)$. If $n \geqslant 2$ then $E / F$ has $a$ relative integral basis.

Proof. Since $E / F$ is unramified (outside $\infty$ ), $D_{E / F}=(1)$. To find $\Delta$, consider the vector space basis for $E / F$ consisting of all subproducts of $\prod_{i=1}^{n} \sqrt{ \pm p_{i}}$. In the determinant expression for $\Delta^{1 / 2}$, each column is obtained by fixing a subproduct and multiplying by a column with entries \pm 1 , corresponding to the action of the Galois group of $E / F$ on the subproduct. We factor out these subproducts from each column and are left with a $2^{n} \times 2^{n}$ matrix consisting of entries $\frac{ \pm 1}{2^{n-2}}$ Since $\sqrt{ \pm p_{i}}$ appears in $2^{n-1}$ subproducts, we find that $\Delta^{1 / 2}=\left(\prod_{i=1}^{n}\left( \pm p_{i}\right)^{2^{n-2}}\right) \cdot m$, where $m$ is a rational integer. Therefore, $\Delta^{-1} D_{E / F}=\left(\Delta^{-1}\right)$ is the square of a principal ideal of $F$, so a relative integral basis exists for $E / F$. Q.E.D.

\section{REFERENCES}

1. E. Artin, Questions de base minimale dans la théorie des nombres algébriques, Colloq. Internat. du Centre National Recherche Scientifique, no. 24, CNRS, Paris, 1950, pp. 19-20; Collected papers of Emil Artin, Addison-Wesley, Reading, Mass., 1965, pp. 229-231. MR 13, 113.

2. G. Fujisaki, Some examples of number fields without relative integral bases, J. Fac. Sci. Univ. Tokyo Sect. IA 21 (1974), 92-95.

3. S. Lang, Algebraic number theory, Addison-Wesley, Reading, Mass., 1970. MR 44 \#181.

4. R. E. MacKenzie and J. Scheuneman, $A$ number field without a relative integral basis, Amer. Math. Monthly 78 (1971), 882-883. MR 44 \#5292.

Department of Mathematics, Stanford University, Stanford, California 94305 